\title{
An Evaluation of the Vulnerable Physician Workforce in the USA During the Coronavirus Disease-19 Pandemic
}

J Gen Intern Med 35(10):3114-6

DOI: $10.1007 / \mathrm{s} 11606-020-05854-7$

(c) Society of General Internal Medicine 2020

\section{BACKGROUND}

The coronavirus disease-19 (COVID-19) pandemic threatens to overwhelm the healthcare resources of the country ${ }^{1}$ and also poses a personal hazard to healthcare workers, including physicians. ${ }^{2}$ Physicians are at an elevated risk of acquiring the disease through exposure to patients who may be symptomatic with the disease or its asymptomatic carriers across the spectrum of clinical specialties. Notably, the physician workforce is not only at risk of losing time spent in clinical care due to these exposures but also at a personal risk from a severe disease that requires hospitalization and is associated with high morbidity and mortality. Notably, physicians 60 years of age and older are at a particularly elevated risk, with $80 \%$ of deaths in China concentrated in this age group. ${ }^{3,4}$ In the early experience in the USA, nearly half of all hospitalizations and intensive care unit admissions and nearly $80 \%$ of deaths have occurred in this age group as well. ${ }^{4}$ To address the potential impact of excluding physicians with a high risk of adverse outcomes based on age, we evaluated the current patterns of age of licensed physicians across the USA.

\section{METHODS}

We used the 2018 database of physicians from the Federation of State Medical Boards (FSMB) that includes all actively licensed physicians across the USA. ${ }^{5}$ The FSMB maintains a database of all licensed physicians in each of the states and US territories compiled during the licensing process, which are sampled biennially and publicly reported. ${ }^{5}$ We extracted publicly available summary data for the physician age in 5-year age bins, nationally and across each of the states. Both at a national and the state level, we used descriptive statistics for the age distribution of physicians and assessed the number and proportion of physicians in each state who would be at elevated risk due to age (age $>60$ years). We used Stata 16 (College Station, TX) for all analyses.

Received March 26, 2020

Accepted April 13, 2020

Published online June 3, 2020

\section{RESULTS}

Of the 985,026 licensed physicians in the USA, 235,857 or $23.9 \%$ were aged $25-40$ years, 447,052 or $45.4 \%$ are 40 60 years, 191,794 or $19.5 \%$ were $60-70$ years, and 106,121 or $10.8 \%$ were 70 years or older. Age was not reported in 4202 or $0.4 \%$ of physicians. Overall, 297,915 or $30.2 \%$ of physicians were 60 years of age or older, 246,167 (25.0\%) 65 years and older, and 106,121 (10.8\%) 70 years or older. States in the USA reported that a median of 5470 licensed physicians (interquartile range [IQR], 2394 to 10,108) were 60 years of age or older. Notably, states of North Dakota $(n=1180)$ and Vermont $(n=1215)$ had the lowest and California $(n=$ $50,786)$ and New York $(n=31,582)$ the highest number of physicians over the age of 60 years (Fig. 1). Across states, the median proportion of physicians aged 60 years and older was $28.9 \%$ (IQR, 27.2\%, 31.4\%) and ranged between $25.9 \%$ for Nebraska and 32.6\% for New Mexico (Fig. 2).

\section{DISCUSSION}

Nearly 1 in 3 licensed physicians in the USA and each of the states are over the age of 60 years, representing nearly 300,000 currently licensed physicians in the USA. The states of California and New York - the two states with the largest outbreaks of COVID-19also have the highest number of physicians 60 years or older.

The study has certain limitations. We do not have information on the specialty expertise of physicians, as some physicians may be more prone to encountering patients with COVID-19. However, as many individuals in the community may be asymptomatic carriers, physicians across specialties are at risk of acquiring the disease as a part of the patient contact during care delivery. Further, we are unable to identify the health status of physicians that may modify their risk or make some younger physicians vulnerable. Finally, physicians may be licensed in more than one state. National estimates, however, represent the number of unique physicians.

In conclusion, older physicians represent a large proportion of the US physician workforce, particularly in states with the worst COVID-19 outbreak. Therefore, their exclusion from patient care will be impractical. Optimizing care practices by limiting direct patient contact of physicians vulnerable to adverse outcomes from COVID-19, potentially by expanding their participation in telehealth may be a strategy to protect them. 


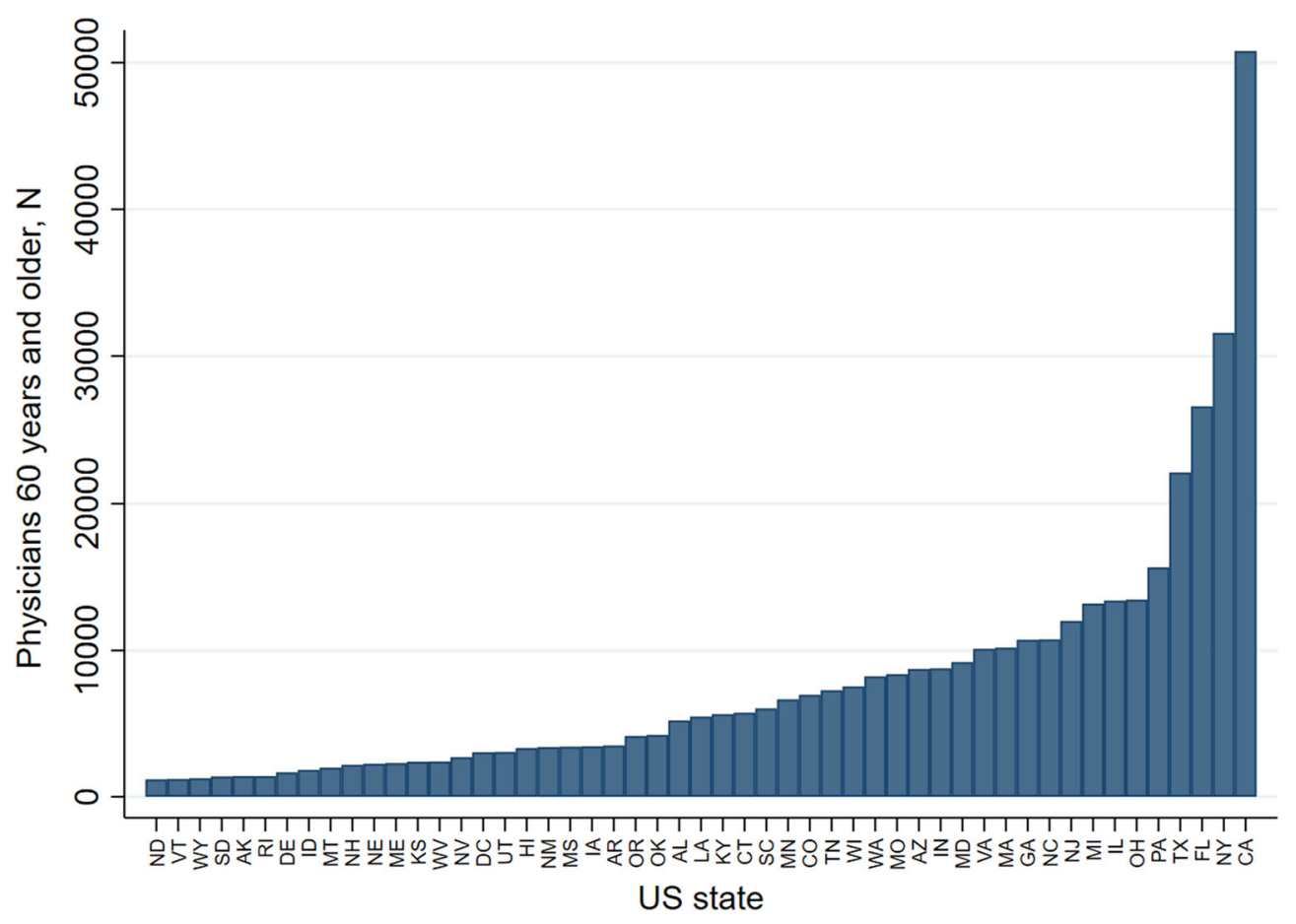

Fig. 1 Number of physicians in each state or US territory 60 years of age and older.

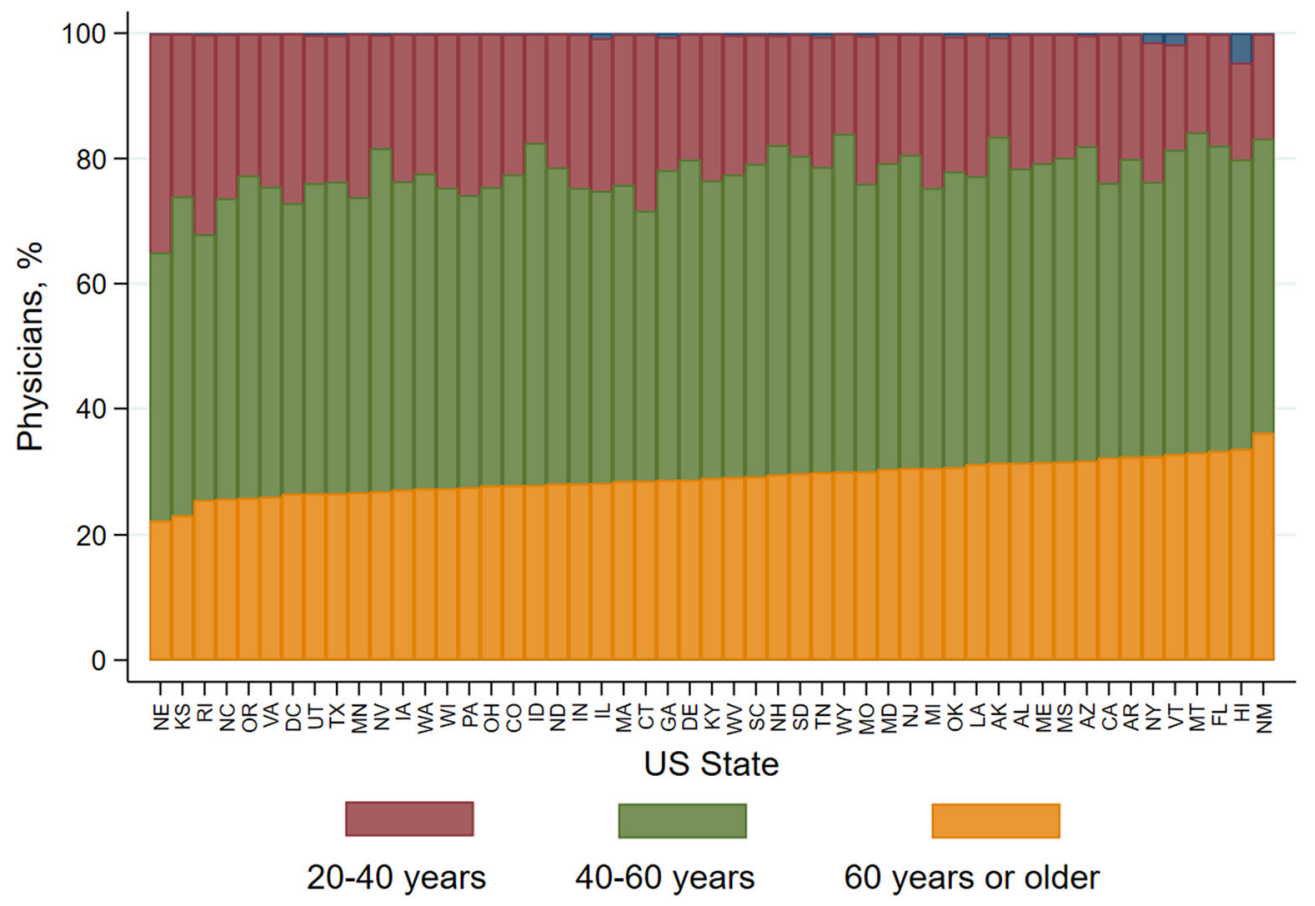

Fig. 2 Proportion of physicians in states and US territories across age groups. The values in blue represent physicians without reported age. 
Rohan Khera, MD MS ${ }^{1}$

Lovedeep Singh Dhingra, MBBS ${ }^{2}$

Snigdha Jain, $M D^{3}$

Harlan M. Krumholz, MD $S M^{4,5,6}$

${ }^{1}$ Department of Internal Medicine, Division of Cardiology, University of Texas Southwestern Medical Center,

Dallas, TX, USA

${ }^{2}$ Department of Medicine, All India Institute of Medical Science,

New Delhi, India

${ }^{3}$ Department of Internal Medicine, Division of Pulmonary and Critical Care, University of Texas Southwestern Medical Center,

Dallas, TX, USA

${ }^{4}$ Center for Outcomes Research and Evaluation, YaleNew Haven Hospital,

New Haven, CT, USA

${ }^{5}$ Department of Internal Medicine, Yale School of Medicine, Section of Cardiovascular Medicine,

New Haven, CT, USA

${ }^{6}$ Department of Health Policy and Management, Yale School of Public Health,

New Haven, CT, USA

Corresponding Author: Rohan Khera, MD MS; Department of Internal Medicine, Division of Cardiology, University of Texas Southwestern Medical Center Dallas, TX, USA (e-mail: rohankhera@outlook.com).

Funding Information Dr. Khera is supported by the National Center for Advancing Translational Sciences (UL1TR001105) of the National Institutes of Health.

\section{Compliance with Ethical Standards:}

Conflict of Interest: Dr. Krumholz works under contract with the Centers for Medicare \& Medicaid Services to develop publicly reported quality measures. He was a recipient of a research grant, through Yale, from Medtronic and the U.S. Food and Drug Administration to develop methods for post-market surveillance of medical devices; is a recipient of a research grant with Medtronic and Johnson \& Johnson, through Yale, to develop methods of clinical trial data sharing; was a recipient of a research agreement, through Yale, from the Shenzhen Center for Health Information for work to advance intelligent disease prevention and health promotion; collaborates with the National Center for Cardiovascular Diseases in Beijing; received payment from the Arnold \& Porter Law Firm for work related to the Sanofi clopidogrel litigation and from the Ben C. Martin Law Firm for work related to the Cook IVC filter litigation; receives payment from the Siegfried \&. Jensen Law Firm for work related to Vioxx litigation; chairs a Cardiac Scientific Advisory Board for UnitedHealth; is a participant/participant representative of the IBM Watson Health Life Sciences Board; is a member of the Advisory Board for Element Science, the Advisory Board for Facebook, and the Physician Advisory Board for Aetna; and is the founder of HugoHealth, a personal health information platform and a co-founder of Refactor Health, an enterprise healthcare AI-augmented data management company. The other authors report no potential conflicts of interest.

Disclaimer: The funder had no role in the design and conduct of the study; collection, management, analysis, and interpretation of the data; preparation, review, or approval of the manuscript; and decision to submit the manuscript for publication.

\section{REFERENCES}

1. Grasselli G, Pesenti A, Cecconi M. Critical Care Utilization for the COVID-19 Outbreak in Lombardy, Italy: Early Experience and Forecast During an Emergency Response. JAMA. 2020;https://doi.org/10.1001/ jama.2020.4031.

2. Livingston E, Bucher K. Coronavirus Disease 2019 (COVID-19) in Italy. JAMA. 2020;https://doi.org/10.1001/jama.2020.4344.

3. Wu Z, McGoogan JM. Characteristics of and Important Lessons From the Coronavirus Disease 2019 (COVID-19) Outbreak in China: Summary of a Report of 72314 Cases From the Chinese Center for Disease Control and Prevention. JAMA. 2020;https://doi.org/10.1001/jama.2020.2648.

4. CDC COVID-19 Response Team. Severe Outcomes Among Patients with Coronavirus Disease 2019 (COVID-19) — United States, February 12March 16, 2020. MMWR Morb Mortal Wkly Rep. March 2020. DOI: https://doi.org/10.15585/mmwr.mm6912e2.

5. Federation of State Medical Boards. Physician Census 2018. Available at: https://www.fsmb.org/physician-census/. Accessed on March 22, 2020.

Publisher's Note: Springer Nature remains neutral with regard to jurisdictional claims in published maps and institutional affiliations. 